\title{
Keeping Pace with the History of Evolving Runtime Models
}

\author{
Lucas Sakizloglou(10凶, Matthias Barkowsky®D, and Holger Giese(1) \\ Hasso Plattner Institute, University of Potsdam, Potsdam, Germany \\ $<$ name>.<surname>@hpi.de
}

\begin{abstract}
Structural runtime models provide a snapshot of the constituents of a system and their state. Capturing the history of runtime models, i.e., previous snapshots, has been shown to be useful for a number of aims. Handling, however, history at runtime poses important challenges to tool support. We present the INTEMPO tool which is based on the ECLIPSE Modeling Framework and encodes runtime models as graphs. Key features of INTEMPO, such as, the integration of temporal requirements into graph queries, the in-memory storage of the model, and a systematic method to contain the model's memory consumption, intend to address issues which seemingly place limitations on the available tool support. INTEMPO offers two operation modes which support both runtime and postmortem application scenarios.
\end{abstract}

Keywords: runtime models $\cdot$ time-awareness $\cdot$ temporal graph queries

\section{Introduction to InTempo}

A (structural) Runtime Model (RTM) provides a snapshot of the constituents of a system and their state [3]. RTMs are typically employed in the context of Selfadaptive Systems (SAS) [4], where a feedback loop adapts the system behavior at runtime in response to external or internal stimuli, the latter represented as model fragments in the RTM and detected via the execution of model queries.

Encoding an RTM as a graph enables detection via graph queries, which specify a sought (graph) pattern. Such an encoding conforms to a metamodel which restricts the structure of model instances and defines types of vertices, edges, and attributes. Formally, these concepts rely on typed, attributed graph transformation [6] where graphs are typed over a type graph.

Capturing the history of RTMs, i.e., previous snapshots, may be useful for a number of aims such as the detection of recurrent behavior or postmortem analysis $[3,8]$. However, handling history at runtime poses important challenges to tool support. Tools are required to enable the specification and timely execution of queries with temporal requirements, i.e., requirements on the evolution of patterns over multiple snapshots. Timely execution is crucial for SAS, where a loop may depend on query results before planning and performing adaptations.

Faced with these challenges, the available tool support is seemingly limited either by the lack of support for direct specification of temporal requirements in graph queries [5] or by the on-disk representation of the model $[8,11]$ that introduces an overhead on execution times in runtime settings, e.g., in SAS.

(C) The Author(s) 2021

E. Guerra and M. Stoelinga (Eds.): FASE 2021, LNCS 12649, pp. 262-268, 2021.

https://doi.org/10.1007/978-3-030-71500-7_13 
We present the InTEMPO (Incremental queries with TEMPoral requirements) tool (available online at [13]) which is based on the eponymous querying scheme in [15] and aims at mitigating these limitations. INTEMPO introduces ITQL, a language for the specification of temporal graph queries, which allow for the expression of temporal requirements. The core functionality of INTEMPO executes a query over an in-memory RTM which captures information about previous snapshots, called Runtime Model with History $\left(\mathrm{RTM}^{\mathrm{H}}\right)$, and returns the pattern occurrences in the $\mathrm{RTM}^{\mathrm{H}}$ that satisfy the specified temporal requirements. INTEMPO is implemented in the ECLIPSE Modeling Framework (EMF) [7] and can be used either via the ECLIPSE user interface or via an API. The latter enables INTEMPO results to be utilized by other tools, e.g., a SAS feedback loop.

INTEMPO offers two operation modes intended for different application scenarios (see Figure 1 for an illustration). The $R T M^{H}$ Analysis (Section 2) constitutes the core functionality of INTEMPO and executes a user-specified ITQL query (in a file with .itql extension-required extensions are in parentheses in Figure 1) over a user-provided $\mathrm{RTM}^{\mathrm{H}}$, i.e., a persisted instance of an EMF model (in the standard $x m i$ format). This mode returns the query results for the given $\mathrm{RTM}^{\mathrm{H}}$. Query results are kept in-between analyses and are updated by each $\mathrm{RTM}^{\mathrm{H}}$ Analysis, which is also known as incremental (query) execution. The $\mathrm{RTM}^{\mathrm{H}}$ Analysis is intended to be used in settings where query results can be further utilized at runtime. For instance, a SAS feedback loop may use INTEMPO to detect problems formulated as patterns, similarly to [9]. Subsequently, the query results may be utilized to plan adaptations which address these problems.

The LogAnalysis operation mode (Section 3) assumes that, instead of being captured by an $\mathrm{RTM}^{\mathrm{H}}$, past and present data about the system have been captured in an event log. INTEMPO introduces E2P, a specification language that allows for the mapping of event types to corresponding modifications of model fragments, i.e, nodes, edges, and attributes. As input, LogAnalysis requires the ITQL query, the log (with comma-separated values), and the E2P mapping. It then processes the log and maintains an internal $\mathrm{RTM}^{\mathrm{H}}$ which it uses to perform $\mathrm{RTM}^{\mathrm{H}}$ Analysis upon every event. LogAnalysis is intended for postmortem scenarios. Thus, it returns the results that were valid after each $\mathrm{RTM}^{\mathrm{H}}$ Analysis sorted by the log timestamps, which affords a global, yet detailed, view on the evolution of the system state.

INTEMPO is capable of containing the data accumulation in the $\mathrm{RTM}^{\mathrm{H}}$ by systematically discovering and discarding data that is obsolete with respect to a given timestamp, i.e., not relevant to future query executions - this capability is presented in detail in [15]. Note that an implicit requirement of both operation modes is that the metamodel of the analyzed system has been encoded as an EMF Ecore model and is available in ECLIPSE (gray input in Figure 1).

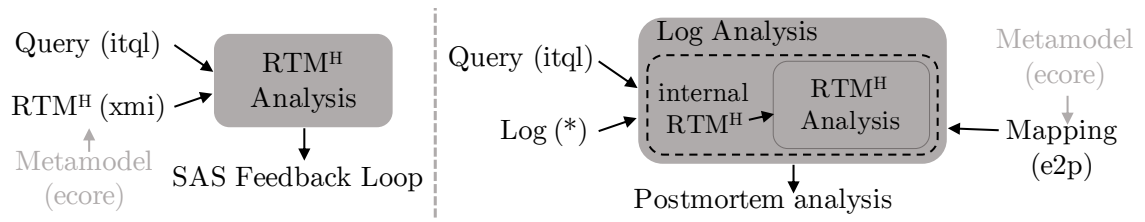

Fig. 1: InTempo Execution Modes and Exemplary Application Scenarios 
Exemplary Application To demonstrate the features and operation of INTEMPO we rely on an example drawn from the case-study conducted in [15]. Based on real-world smart medical environments, the case-study envisions a Smart Healthcare System (SHS) where

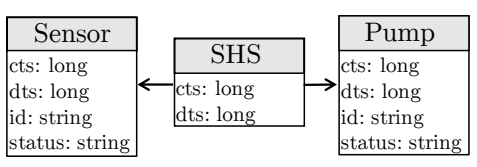

Fig. 2: SHS Metamodel certain medical procedures are automated and performed by devices, such as a smart pump administering medicine or a sensor tracking patient data and diagnoses - as otherwise a clinician would be doing. Data collected from the SHS are aggregated and recorded in medical (event) logs.

INTEMPO requires a metamodel which has been instrumented such that all nodes have at least two attributes named cts and dts, which capture the time point of creation, respectively deletion, of the node in the system. As an example, see the metamodel of our SHS in Figure 2. Note that to encode cts and dts for edges in EMF, the respective edges would have to be modeled as nodes. Technically, an RTM ${ }^{\mathrm{H}}$ is an instance of such a metamodel. See $\mathrm{G}_{3}$ in Figure 3 for an example based on the SHS metamodel: The $\mathrm{RTM}^{\mathrm{H}}$ reflects that a node of type Sensor that is attached to the patient with $i d=1$ has been activated and thus has been added to the SHS at timestamp 3. The sensor status reflects that the patient has been diagnosed with sepsis. The value $\infty$ reflects that a dts for this node has not been set, i.e., the node is still present in the modeled system.

\section{RTM $^{\mathrm{H}}$ Analysis}

This section presents an exemplary query in ITQL which it then uses to demonstrate the $\mathrm{RTM}^{\mathrm{H}}$ Analysis. It concludes with technical details.

InTempo Query Language (ITQL) Formally, a temporal graph query $q$ is characterized by a (graph) pattern $p$ and an application condition ac, denoted $q=(p, a c)$. A match $m$ corresponds to an occurrence of $p$ in the $\mathrm{RTM}^{\mathrm{H}}$. In order for $m$ to be valid, it must satisfy the $a c$. ITQL supports the formulation of $a c$ in the Metric Temporal Graph Logic (MTGL) [10] which supports operators such as negation $(\neg)$, existential quantification $(\exists)$, conjunction $(\wedge)$, and the metric, i.e, interval-based, temporal operators until $\left(\mathrm{U}_{I}\right.$, where $I$ is a time interval over $\left.\mathbb{R}_{0}^{+}\right)$and since $\left(\mathrm{S}_{I}\right)$, as well as abbreviations such as eventually, i.e., $\diamond_{I} \exists n=$ true $\mathrm{U}_{I} \exists n$, where $n$ is a graph pattern and true is always satisfied. MTGL also supports the nesting of patterns to bind graph elements in outer conditions and relate them to inner (nested) conditions, i.e., elements common to two patterns $n_{1}$ and $n_{2}$ refer to the same element in the $\mathrm{RTM}^{\mathrm{H}}$.

MTGL is able to express real-time properties such as "every patient diagnosed with sepsis, must eventually within 5 time units be given the proper drug" (adjusted from the medical guideline in [14]). In an $\mathrm{RTM}^{\mathrm{H}}$ of the SHS, INTEMPO can find violations of the property above by executing the ITQL query $q_{1}=\left(n_{1}, \kappa\right)$, with $\kappa$ the MTGL formula $\neg\left(\diamond_{[0,5]} \exists n_{2}\right)$ and $n_{1}, n_{2}$ patterns representing a sepsis diagnosis and drug administration respectively. The query searches for matches of $n_{1}$ in the $\mathrm{RTM}^{\mathrm{H}}$ that satisfy $\kappa$, i.e., for patients that, 


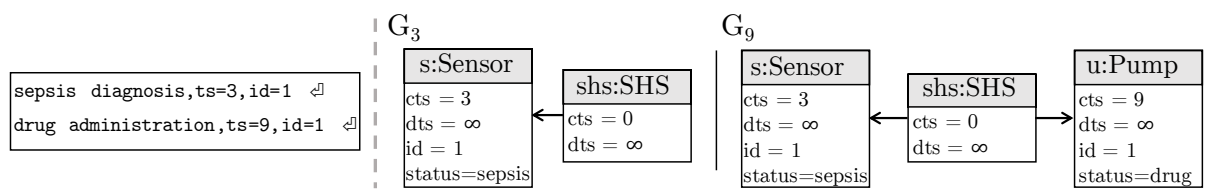

Fig. 3: Exemplary Medical Log and Corresponding $\mathrm{RTM}^{\mathrm{H}} \mathrm{G}_{3}$ and $\mathrm{G}_{9}$

although diagnosed with sepsis, did not receive a drug within the designated time. In INTEMPO, each match is associated with a temporal validity, i.e., a set of time intervals for which, based on the overlap among the cts and dts of the matched elements and the interval for which $a c$ is satisfied, the match is valid. ITQL also allows for the definition of OCL constraints [12] on sought patterns.

Output The ITQL specification for the query $q_{1}$ is shown in Figure 4 . Performing $\mathrm{RTM}^{\mathrm{H}}$ Analysis for the query $q_{1}$ on the $\mathrm{RTM}^{\mathrm{H}}$ $\mathrm{G}_{9}$ of Figure 3 returns one match, since there is indeed no Pump attached to the SHS, i.e., a match for $n_{2}$, within five time units after a Sensor was activated, i.e., a match for $n_{1}$ was found. The temporal validity interval $[3,4]$ is returned together with the match. The match, i.e., violation, is indeed valid only for that interval since after timestamp 4, a match for $n_{2}$ starts to exist within five time units of a match for $n_{1}$. If the API of INTEMPO is used, the

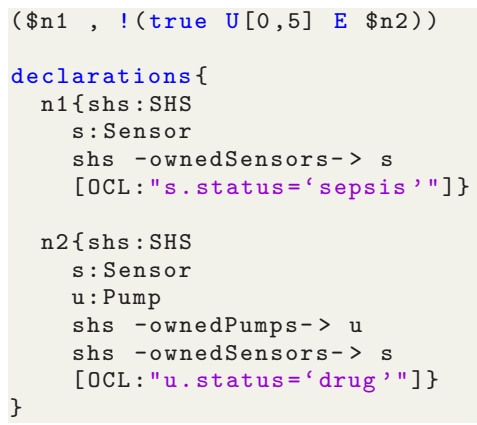

Fig. 4: Example query in ITQL query returns the match of the $n_{1}$ pattern, i.e., the EMF objects, together with the temporal validity. In case INTEMPO is used via the UI it displays a message box in ECLIPSE with the following message: SHS@0[] Sensor@3 [status=sepsis] $[[3,4]]$. Note that "@" precedes the cts of an object and values within square brackets are attributes of the object.

Technical Details For the execution of temporal graph queries, InTEMPo employs the operationalization framework presented in [15]. The framework supports the decomposition of a query into a suitable ordering of simpler sub-queries which is executed bottom-up. The outermost query computes the overall result. For pattern-matching, INTEMPO employs the Story Diagram Interpreter from [1] which uses heuristics shown to reduce the pattern-matching effort. INTEMPO provides an XTEXT [2] editor for ITQL which supports completion suggestions for element types and validation of the query syntax.

\section{LogAnalysis}

This section demonstrates the LogAnalysis operation mode which assumes that data from past states have been captured as events in a log. INTEMPO offers the capability to process the system changes and, upon each change, obtain an updated $\mathrm{RTM}^{\mathrm{H}}$ which is then used internally to perform $\mathrm{RTM}^{\mathrm{H}}$ Analysis. 


\section{Events-to-Patterns (E2P) Spec-} ification Language The mapping of $\log$ events (which encapsulate system changes) to prescribed modifications on an $\mathrm{RTM}^{\mathrm{H}}$ is facilitated by E2P. An E2P specification consists of mappings between events and actions that should be performed on an $\mathrm{RTM}^{\mathrm{H}}$. E2P supports five actions

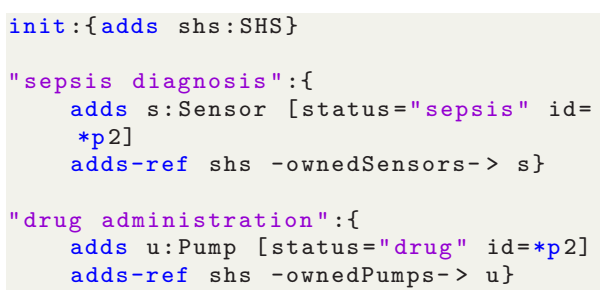

Fig. 5: E2P Example for SHS (formulated as verbs): adds, to add a node and optionally assign values to the added node's attributes; adds-ref, to add an edge between two nodes; modifies, to modify the attribute values of a node; deletes and deletes-ref, to delete a node, respectively an edge, from the $\mathrm{RTM}^{\mathrm{H}}$. To accommodate linked data, E2P allows for the indexing of added nodes so that later events can refer to modifications that have been processed earlier. An example of an E2P mapping from an exemplary log in Figure 3 (left) to the corresponding elements of the SHS is shown in Figure 5. Note that edge types, e.g. OwnedPumps, are not depicted in Figure 3.

As an example, the event drug administration from the medical log in Figure 3 corresponds to the following changes to the (internal) $\mathrm{RTM}^{\mathrm{H}}$ : a Pump is created; its attribute status is set to "drug" and its attribute id takes the value of the second field after the event name (expressed by the special $* p$ token), i.e., the id field in the $\log$ of Figure 3. By default, the cts is set to the value of the event field that is next to the event name, i.e., the ts field in Figure 3. The init statement is used to initialize the $\mathrm{RTM}^{\mathrm{H}}$ and the cts of nodes within is set to zero. To increase the readability of specifications, an explicit assignment for the dts may be omitted: Unless there is an attribute assignment, the dts of all nodes is set to the maximum value supported.

Output LogAnalysis provides a view on the matches per event timestamp. Performing $\log$ Analysis on the query $q_{1}$ and the $\log$ of Figure 3 would return:

\section{@3 SHS@O[] Sensor@3[status=sepsis] [ $[3, \infty]]$ \\ @9 SHS@0[] Sensor@3[status=sepsis] [ $[3,4]]$}

First, the sepsis diagnosis event is processed which makes the internal $\mathrm{RTM}^{\mathrm{H}}$ be identical to $\mathrm{G}_{3}$ in the same figure. The query is executed using $\mathrm{RTM}^{\mathrm{H}}$ Analysis and returns a match, i.e., violation, since at that moment a match for $n_{2}$ does not exist in the graph. The temporal validity is equal to $[3, \infty]$, i.e., the match is valid from time point 3 onward. Next, the drug administration event is processed which leads to $\mathrm{G}_{9}$. The result of $\mathrm{RTM}^{\mathrm{H}}$ Analysis for $\mathrm{G}_{9}$ is the same as the result described in Section 2.

Technical Details In LogAnalysis the query execution framework monitors the $\mathrm{RTM}^{\mathrm{H}}$ for changes and, upon every change, recomputes the matches. Previous matches are kept in-between executions and therefore the query is executed incrementally. Similarly to ITQL, E2P is supported by an XTEXT editor that offers syntax validation and completion suggestions for element types. 


\section{Conclusion and Future Work}

We presented INTEMPO, an EMF tool which enables the specification and incremental execution of temporal graph queries over a runtime model with history. The latter can be either provided as input or obtained by an event log. INTEMPO stands out from relevant tools owing to the integration of temporal requirements into graph queries, the in-memory representation of the model, and the systematic measures to contain memory consumption despite the accumulation of temporal data. Moreover, INTEMPO offers input editors with features that aim at helping the user, e.g. syntax validation. In the future, besides streamlining INTEMPO, we plan to perform extensive evaluation and comparisons with other tools. Moreover, we plan to explore the utilization of INTEMPO in self-adaptation scenarios where the history of the system is required.

\section{References}

1. Barkowsky, M., Giese, H.: Hybrid search plan generation for generalized graph pattern matching. JLAMP 114, 100563 (2020)

2. Bettini, L.: Implementing domain-specific languages with Xtext and Xtend. Packt Publishing Ltd (2016)

3. Bencomo N., Goetz S., and Song H.: Models@ run. time: a guided tour of the state of the art and research challenges. SoSyM 18.5 (2019)

4. Brun, Y., Di Marzo Serugendo, G., Gacek, C., Giese, et al.:Software engineering for self-adaptive systems, pp. 48-70. Heidelberg (2009) Springer

5. Búr, M., Szilágyi, G., Vörös, A., Varró, D.: Distributed graph queries over models@run.time for runtime monitoring of cyber-physical systems. STTT 22(1)

6. Ehrig, H., Prange, U., Taentzer, G.: Fundamental Theory for Typed Attributed Graph Transformation. ICGT Berlin, Heidelberg (2004) Springer

7. Eclipse Foundation: Eclipse modeling framework (EMF) (Aug 2020), https://www. eclipse.org/modeling/emf/, accessed: 2020-10-11

8. García-Domínguez, A., Bencomo, N., Parra-Ullauri, J.M., García-Paucar, L.H.: Querying and Annotating Model Histories with Time-Aware Patterns. MODELS. pp. 194-204 (2019) ACM/IEEE

9. Ghahremani, S., Giese, H., Vogel, T.: Efficient utility-driven self-healing employing adaptation rules for large dynamic architectures. ICAC (2017)

10. Giese, H., Maximova, M., Sakizloglou, L., Schneider, S.: Metric Temporal Graph Logic over Typed Attributed Graphs. FASE, (2019) Springer

11. Gómez, A., Cabot, J., Wimmer, M.: TemporalEMF: A temporal metamodeling framework. ER, vol. 11157, pp. 365-381. (2018) Springer

12. Kleppe, A., Warmer, J.: An introduction to the object constraint language (OCL). In: TOOLS p. 456 (2000)

13. MDELab: InTempo Homepage, http://www.hpi.uni-potsdam.de/giese/public/ mdelab/mdelab-projects/intempo/, accessed: 2021-01-19

14. Rhodes, A., Evans, L.E., Alhazzani, W., Levy, M.M., et al.: Surviving sepsis campaign: International guidelines for management of sepsis and septic shock: 2016 . Intensive care medicine 43(3), 304-377 (2017)

15. Sakizloglou, L., Ghahremani, S., Barkowsky, M., Giese, H.: A scalable querying scheme for memory-efficient runtime models with history. MoDELS pp. 175-186. (2020) ACM/IEEE 
Open Access This chapter is licensed under the terms of the Creative Commons Attribution 4.0 International License (http://creativecommons.org/licenses/by/4.0/), which permits use, sharing, adaptation, distribution and reproduction in any medium or format, as long as you give appropriate credit to the original author(s) and the source, provide a link to the Creative Commons license and indicate if changes were made.

The images or other third party material in this chapter are included in the chapter's Creative Commons license, unless indicated otherwise in a credit line to the material. If material is not included in the chapter's Creative Commons license and your intended use is not permitted by statutory regulation or exceeds the permitted use, you will need to obtain permission directly from the copyright holder. 